$\square: \quad L e$ sourire des ruines - à propos de l'ouvrage de Jacques Réda

\title{
PatRick Baudry
}

Professor de Sociologia da Universidade de

Bordeaux (França) 
. 
Trois approches de la notion de ruine peuvent être distinguées. Dans la première, c'est un événement catastrophique qui la produit. Elle tient alors de l'effondrement. Elle s'apparente à un accident dans le cours d'une histoire. Elle appelle la reconstruction. Selon cette conception, la ruine accidentelle est une rupture. Mais en tant que telle, elle peut servir à légitimer la vision de l'histoire comme processus : la ruine serait un arrêt momentané dans le cours d'un progrès dont le bien-fondé et l'évidence ne sauraient être remis en cause. Eventuellement le territoire éventré ne fait pas l'objet d'une reconstruction : on y pose une dalle pour que l'irréparable soit commémoré. Dans la seconde, elle devient objet du passé, vestige, témoignage d'un autrefois prestigieux. Elle ne s'efface pas mais se conserve. Elle orne la ville, décore le territoire, fait oeuvre de mémoire. Elle peut donc faire l'objet d'une esthétisation. Dans la troisième, la ruine doit se comprendre comme mouvement de déracinement qui place le sujet humain au cœur du travail de symbolisation. Elle n'est pas seulement le produit d'un événement, mais un processus qui traverse l'expérience même de l'habitation.

Il existe des gens qui font de la ville un savoir. Ils la parcourent parce qu'ils entendent la dominer. Ils prétendent être en leur territoire. Il en est d'autres qui devinent les gouffres, la trouée du monde, l'aventure subjective et "l'anthropologie de soi" (Jean Duvignaud') qu'il faut inventer pour raconter la parole décousue, les bribes de vie, les moments d'existence, le tremblement des détails.

Les nazis voulaient que leur ordre demeure encore dans sa propre mise en ruine. L'ordre nazi pourrait être ruiné, mais ses ruines porteraient encore la trace indélébile de cet objectif pur, sans erreur, sans faille. Les ruines seraient, à défaut de victoire, le nazisme continué, définitivement marqué dans le sol. Mais "les Ruines", les Ruines de Paris de Jacques Réda ${ }^{2}$, ne conservent aucune trace, elles n'ont pas de docilité historienne. La ville où l'on passe ne va pas, ordinairement, sans ruine du monde. La ruine procure cette hésitation confiante qui fait être à la permanence possible d'une vie fragile.
'Voir Jean Duvignaud Le sous texte, Arles, Actes Sud, 2005.

${ }^{2}$ Voir Jacques Réda, Les ruines de Pars, Paris, Gallimard, 1977. 
${ }^{3}$ Voir Henri-Pierre Jeudy Le Désir de catastrophe, Paris, Aubier, 1990.

\section{Le hors lieu}

Les Ruines de Paris n'évoquent pas le temps qui ronge les constructions, le climat d'abandon qui domine un monde défait ou l'aspect dérisoire de ce qui reste des habitations ou des loisirs d'une société promise à la décrépitude. Les Ruines installent un écart entre l'image que voudrait donner le bâti avec tout son pouvoir d'aménagement et le rapport trouble que le décor esthétique lui-même finit par diffuser. Cette façade fière, cette modernité efficace, croient-elles qu'elles pourraient demeurer intactes? Il semble plutôt que le neuf, de toute sa force, contienne un vieillissement accéléré, que sa perfection attende une atteinte: la venue des Ruines qu'aucune culture ne songerait à supprimer. L'idée de ruines, du moins, attise le sentiment (presque la certitude) que ce mouvement existe.

Nulle pente morbide ici. Aucun sentiment qui devrait faire l'objet d'un soin. L'homme qui vit avec le mouvement des Ruines n'est pas victime de mauvaise humeur. Il ne rêve ni d'être individu hors du monde ni de s'y confondre. Il assiste à ce qui lui appartient sans souci d'en être propriétaire. L'homme ou la femme des Ruines sont fondamentalement de ce monde sans qu'ils aient besoin d'être en leur territoire. L'étrangeté de toute situation n'est plus alors une pathologie ${ }^{3}$. L'existence qui retranche de la vie fait être aux mouvements instables. C'est cette instabilité et cette ambiguité qui sont plus précieuses qu'aucun retrait superbe ou qu'aucune force de terrain. Nul besoin donc de précipiter la décomposition d'un "corps" de ville, d'espérer que le mur se craquelle ou que la fissure se généralise. C'est plus simplement la fixité du temps et la dureté de l'être qui paraissent comme des naïvetés sans attrait. C'est une inquiétude presque sereine qu'éprouve Jacques Réda, comme s'il fallait souhaiter que l'épaisseur du temps fasse place enfin au vide et qu'il en puisse accueillir la complicité.

Jacques Réda ne se désespère pas d'un monde qui lui manquerait ou qui, par ses fuites, aurait trahi ses attentes: il dit qu'il est "adossé aux ruines de Paris" (p.73). Loin de la 
capitale, il s'y trouve encore "adossé". A Dijon ou à Brest, n'importe où visiblement, il est "adossé aux ruines". Pourquoi ? Parce que la ville (Paris ou n'importe quelle autre) n'est jamais seulement cette ville. Je devine que Réda n'a jamais cru à la province ou à la banlieue comme s'il s'agissait de lieux: ce sont des tournures qui emportent les directions, ce sont des ambiances qui décentrent les endroits, ce sont des exils dont on profite partout.

N'est-ce pas le tourment qui nous assaille lorsque nous visitons pour la première fois et pour un trop court séjour une ville: n'en voir que les jolies choses, que les choses à voir, mais sans avoir pu faire venir les ruines? Sauf que depuis la ville que nous habitons ailleurs, et dans laquelle nous sommes toujours ailleurs, les Ruines peuvent venir dans la place imposante, dans les façades remaniées et même jusqu'à cette poignée de porte cossue. L'ahurissement que procurent les Ruines donne l'intelligence de confondre les lieux. L'important n'est pas de ne plus savoir où l'on est mais de n'avoir plus besoin de le savoir. Le lieu confondu n'est pas pris pour un autre. Il s'agit de le confondre, comme on le dit d'un témoin trop sûr de ses savoirs tout personnels. L'important est que le lieu soit délogé de sa superbe territoriale, mis en mouvement depuis notre immobilité.

Avec les Ruines, la ville n'apparaît plus comme ce corps plein qui contiendrait tous ses liens, tous ses tissus, tous ses mécanismes vivants. La ville n'est pas un corps. Elle est surtout un hasard. Ou bien nous ne pourrions pas l'habiter un peu, ni nous laisser imbiber par ses sonorités. Même si nous y sommes, nous venons à la ville. Et il se peut, qu'en y habitant depuis toujours, celle-ci se retire. La ville arrive comme une ondée, comme si ce jeu semblait venir d'elle et que nous devrions croire qu'elle existe. Mais nous n'y croyons jamais tout à fait. D'où le jeu de Réda : guetter le creux, la torsion des lignes, ces endroits où les rails renoncent à avancer, tout en même temps que le voyage est là : dans cette avancée indécise des boulevards vides, dans ces piles de bois, dans ces boutiques ou encore depuis le dos de cette femme qui passe sans savoir le mouvement de ses cheveux. 
Les Ruines, oui, et il faut donc un pluriel pour mettre en mots ces bizarreries. Elles révèlent le lyrisme des rues sans histoire aussi bien qu'elles épousent leur petit côté. Avec les Ruines, c'est aussi l'écriture qui s'exige: seul moyen, sans aucune espérance, d'avoir talent de s'égarer.

L'ouvrage de Jacques Réda m'étonne. Plus je le compulse comme un guide impossible, comme une histoire sans histoire, comme un récit sans récit, et plus ce livre que j'ouvre à n'importe quelle page pour retrouver la page qui importerait peut-être, m'intrigue. Pourquoi donc parle-t-il de ce que j'ai si bien connu, lors même que ses descriptions marquent une époque qui ne fut pas la mienne? Réda était donc passé par tous ces endroits sans intérêt que j'ai tant pratiqués. Ainsi la rue Balard où, pour ma part, je guettais le sud, l'arrivée du sud avec précipitation de l'océan par grandes houles. Ou encore cette rue des Pyrénées où je connaissais une femme qui vivait nue, et aussi la rue de Vaugirard. Près du Sénat, la longue rue prendrait peut-être une importance monumentale, mais ailleurs elle ne vaut rien, sauf un carrefour, un rideau de fer, une interdiction de stationner, des trottoirs. La banlieue est toujours dans la ville. Elle est son échappée. Son décalage heureux, même sous des airs ternes. Comme Réda, j'aime le jazz et donc la pluie, les arrêts de bus où l'on s'ennuie absolument, les quais déserts, les ruelles sombres. Comme moi, il dit sa passion pour les boutiques, les choses que l'on touche, les objets qu'on achète comme si l'on attrapait un trésor et aussi la fatigue qui épuise tout le corps rien qu'à l'idée d'aller acheter du pain (p.40). A force de lire ce livre diagonal et surtout décentré, je me suis demandé si je n'étais pas moi-même Jacques Réda. Et que j'aie pu oublier avoir changé de nom un jour, peut-être parce qu'il aurait fallu que j'abandonne à cet homme que je n'ai jamais vu cette faculté d'avoir été moi-même en ne le sachant pas. Risible élucubration. Bien sûr un effet des Ruines : leur sortilège même. Cette ville et ses dehors (mais qui sont ses dedans, la banlieue dans la ville dévissée) dont Réda parle, c'est à la fois cette ville matérielle, objective, concrète et ce que la ville nous fait: quand notre intériorité s'éprouve comme 
externe, quand le monde présent diffuse une préhistoire qui nous taraude et à laquelle, définitivement, nous cherchons obstinément à ne comprendre rien. "L'intériorité ", par exemple, que peut-elle être pour qui ne croit à aucune évasion ${ }^{4}$ ?

Une certaine idiotie, je crois, me rapproche aussi de Réda ${ }^{5}$. Capable de camoufler son désarroi aux passagers d'une gare sur le quai d'en face et (peut-être en même temps) de sourire parce que le soleil vient entre ses doigts, ou parce que le ciel est noir et que la ville est alors "aquatique" (p.81). Réda ne peut être qu'ahuri. Il dit bien qu'il ne sait pas lui-même s'il est gai ou triste, s'il chemine vers une énigme ou une signification (p.114). A la même page, il dit bien qu'il ne "cherche pas trop à comprendre".

L'écriture de Réda ne vise pas par un style à témoigner d'émotions. L'auteur ne cherche pas à faire part de ses impressions. Encore moins ferait-il le guide ou le savant, le mémorialiste érudit ou le conteur exemplaire. L'écriture de Réda ne sert pas une habilité de description. Il s'agit dans l'expérience des mots qui se bousculent, s'appellent et se distancient, s'allument et s'éteignent, de dire une présence des Ruines sans souci de reportage. Réda ne rapporte rien. Il se place, "adossé aux ruines", comme ces clichés, ces images simples, ces souvenirs conservés qui ne serviront jamais de souvenirs. Il avance dans une mémoire inutile, il recule devant les rails qui se tordent et qui l'aimantent. Il part dans un talus, descend un escalier, rencontre ses tocades, s'en moque, erre encore, navigue près d'un bac à sable, raconte que les habitants ne sont pas seulement des semblables, mais des étrangers et surtout des inconnus.

\section{L'image invisible}

Mettre en images le monde, est-ce le devoir de l'exercice littéraire? Rajouter des images à une vie qui, naturellement, en manquerait, est-ce la tache de la poésie? Avec Réda, on comprend que l'habileté est presque inverse. Il s'agit peu de révéler les images d'un monde qui nous donnerait d'abord son opacité. Il faut plutôt diminuer les
+ Voir Emmanuel Lévinas De L'évasion, Montpellier, Fata Morgana, 1982.

${ }^{5}$ Voir Patrick Baudry " La chronique comme une aventure " in $\mathrm{La}$ Chronique dans tous ses états, sous la direction d'HenriPierre Jeudy, Paris, Sens \& Tonka, 2004, p.87. 
scintillances qui s'imposent. Il faut, bizarrement peut-être, rendre à l'obscurité son astuce voyante. Il faut ainsi rendre à l'image invisible son pouvoir de nous subjuguer: parce que son insoumission nous gagne et que nous voulons bien jouer alors avec elle.

Jacques Réda qui "écrit si bien" comme on pourrait le dire bêtement, sait qu'il veut échapper à la description ou qu'il n'en a pas le pouvoir plein et entier. Dans l'espèce de retrait involontaire où il se tient, c'est un lieu introuvable qu'il raconte sans cesse. Il écrit: "Peut-être que si je réussissais enfin à tout décrire, à l'instant même où le moindre brin d'herbe ou de fil de fer paraît, je comprendrais quel rôle ambulant je tiens moi dans ce rythme [...]. Mais je ne suis qu'un des points qui fuient à l'intersection des deux courbes, que dessinent en ogive les rails et le soleil qui décroît" (p.141, 142).

Jacques Réda aurait pu témoigner de la rue mise en travaux, du vieil immeuble désossé, de la façade éventrée, des lieux arides, des endroits à l'abandon, des coins morts. Sans doute découvre-t-il quelques maisons que l'on détruit. Mais s'il a la curiosité d'y trouver des objets et l'idée d'en conserver quelques uns, il se ravise: ces fenêtres mises par terre sont peut-être intactes, mais il comprend qu'elles sont inutilisables. En parlant d'objets plus intimes (cartes postales, cahiers d'école, vieux jouets), il dit bien que remporter tout cela pour le descendre dans sa cave serait "encore plus navrant” (p.19). On devine bien que Réda n'aime guère la modernité architecturale et l'on pourrait comprendre qu'il milite pour le quartier à l'ancienne, pour l'atmosphère vivante de la ville d'autrefois ou le charme des vieilles rues. La modernité d'un hôtel flambant neuf, l'originalité sans précédent d'un ascenseur extérieur qui monte et qui descend sur la façade, Réda les associe à un style certes "éternel" mais aussi "mortuaire très égyptien" (p.39). Plus loin, il parle encore du "luxe funéraire" des immeubles du Front de Seine (p.94). Il précise que les constructions ultra-modernes "résisteront moins bien aux âges que les tombeaux". Puis il affirme: "Et ce qui d'abord disparaîtra, c'est l'ascenseur extérieur qui monte et qui descend comme une bulle" (p.39). 
Oui, sans doute Jacques Réda semble-t-il avoir quelque prédilection pour les lieux désertiques. Après les maisons démolies, puis les casernements désaffectés, ce sont des abords de gare, des rails déterrées, des tunnels qui ne mènent plus nulle part et qui ne voient passer aucun train qu'il raconte. Mais l'essentiel ne tient ni à ce qu'il voit ni à ce qu'il serait pour le voir. Il est peu question d'identité, de recherche de soi dans l'épreuve d'une errance, d'introspection au moyen d'un laborieux cheminement. Réda ne fait pas le bilan de son existence comme s'il fallait maîtriser un tant soit peu sa propre histoire et tenter de devenir savant. Ce n'est pas sa pathétique solitude qu'il guette en reflet dans des ruines. Qu'il s'agisse de constructions nouvelles ou anciennes, d'édifications ou de démolitions, d'endroits peu fréquentés ou de lieux de foule, ce qui inquiète sereinement Réda c'est le décalage que le mouvement des ruines impulse. Aussi bien n'est-il jamais question de désappointement ou de déception devant un monde sans prise. Jacques Réda n'est pas effaré par l'indifférence d'un décor qui, quoique changeant, resterait immuable. Il n'est pas cet être fixé devant un horizon. Le décalage à quoi il tient et qu'il aime rejouer, n'a besoin ni du drame ni de la fête. Il ne nécessite aucune occasion. C'est ordinairement que le mouvement de ce décalage ${ }^{6}$ s'agence dans une dimension énigmatique. C'est la relance de cette énigme, traversant de toutes parts un territoire, qui l'exalte sans besoin d'espérance: "Sans doute ce qui m'attend toujours au bout reste proche de la déception, mais cette déceptionlà m'exalte, me simplifie, me transforme comme religieusement en pure attente de rien" (p.156).

La maladresse, par exemple, n'est plus alors un défaut de structuration des liens sociaux, mais une disposition. En étant légèrement à côté de la plaque, en expérimentant l'incongruité d'être là, Jacques Réda se sait "indécis" et se dit dans une formule bizarre "entre oubli et peut-être, passant" (p.137). Ce n'est pas un tempérament qui est à l'œuvre, une qualité ou un défaut qui l'accable. C'est un rapport au monde qui s'exprime. C'est le monde même qu'il s'agit de révéler dans ses troubles et dans ses jeux. C'est au fond un sérieux qui se récuse, un
${ }^{6}$ Voir Patrick Baudry Violences invisibles, Bègles, Editions du Passant, 2004, p. 138. 
${ }^{7}$ Emmanuel Lévinas

Ethique comme philosophie première, Paris, Payot \& Rivages, 1998, p.105.

" Emmanuel Lévinas, idem, p.87, 88. positionnement sûr qui est refusé. Réda n'a nul besoin de preuves pour montrer la fausseté de l'exactitude. "Nulle part du reste, je ne m'attarde; j'imagine tout de suite être ailleurs", écrit-il (p.169).

Je pense alors à Emmanuel Lévinas quand il dit: "moi, je m’interroge déjà si mon être est justifié, si le $D a$ de mon Dasein n'est pas déjà l'usurpation de la place de quelqu'un"7. Lévinas parle ainsi de la "Réserve du noninvesti, du non-justifié, de "l'étranger sur la terre", selon l'expression du psalmiste, du sans patrie ou du "sans domicile" qui n'ose pas entrer" .

Faut-il croire que notre devoir est de traverser la ville en long et en large, comme si nous étions condamnés à quelque pathétique occupation ou comme si nous devions croire que nous dominons "les choses"? Sans doute subissons nous ces astreintes à arpenter, calculer, et tout de même savoir un peu qui nous sommes. Reste qu'il n'y a pas à choisir entre bonheur ou malheur, carences psychologiques ou pleine santé, humeur maussade ou voix enjouée. Il ne suffit pas non plus de décider que la vie est fabuleuse ou navrante. "Parfois j'ai tout rebâti en proie à de l'espérance rétroactive, d'autres fois tout écrasé sous l'évidence du désespoir", écrit Jacques Réda (p.170).

Mais l'essentiel, qui n'est pas « essentiel ", c'est le travers du monde et de l'homme, quand les choses avec lesquelles on est vont elles aussi de travers.

Ne pas trop expliquer. Sourire avec candeur. Avoir peur parfois. Se laisser aller aux mouvements du train, le front collé à la vitre, l'œil guettant partout et le regard se rencontrant dans son reflet. Avoir éventuellement l'air d'un crétin, ou peut-être donner malgré soi le message d'une exaltation un peu sotte. Non pas tellement croire que tout s'arrangera, que tout finira bien par s'arranger, mais donner parfois acte à la confiance, lors même qu'on aurait rien à dire aux autres gens.

"Tandis que j'écrivais, imitant de vagues équations sur ma page de gauche pour ne pas intriguer deux ingénieurs, l'obscurité s'établissait, et pas trace d'un astre. Si le train s'éteignait on verrait certainement de très loin, dans la campagne, l'amour qui me traverse de sa lueur" (p.145). 